\title{
Study on Comparative Analysis of Camera Calibration
}

\author{
*Tae-Eun Kim, Department of Multimedia, Namseoul University, 91 Daehak-ro Seonghwan-eup Seobuk-gu \\ Cheonan-si Chungnam, 31020, South Korea, tekim5@empas.com \\ ${ }^{*}$ Corresponding Author
}

\begin{abstract}
Several error factors exist when restoring three-dimensional images from two-dimensional images acquired by cameras. In particular, studies that interpret error factors from camera parameters are critical to stereo convergence and three-dimensional image restoration performance. Several error factors exist when restoring three-dimensional images from two-dimensional images acquired by cameras. In particular, in order to interpret the error factors arising from the camera parameters, the camera inspection was performed by applying the methods of calibration of the Tsai camera and the Direct Linear Transformation (DLT) camera calibration using various images entered by the camera. The camera inspection was carried out by applying the method of calibration of the Tsai camera and the method of calibration of the DLT camera. The performance of each camera calibration method was compared by presenting the results of evaluating stereo convergence and restoration performance using camera parameters obtained through camera tests. Modeling techniques for the camera test process were presented through this study. In the future, it is believed that these findings will be used as basic data for image information correction when obtaining image information from the imaging system.
\end{abstract}

Keywords: Camera Calibration, Tsai Calibration Method, Three-dimensional Images, Camera Parameter, Direct Linear Transformation, Computer Vision

Received: 07.12.2020 $\quad$ Accepted: 13.01.2021 $\quad$ Published: 03.02.2021

\section{INTRODUCTION}

Camera calibration is to determine spatial pose and positional relation of camera in the process of determining geometrical relationship between any image point and any point on 3D spatial coordinates, and to calibrate image distortion arising from this process, which is a very important issue in the field of computerized visual recognition. Only when these camera parameters are accurately calibrated, an accurate 3D real position can be obtained from the image coordinates, and conversely, 2D image coordinates can be accurately estimated from 3D real coordinate[1,2].

As a basic research for the development of 3D TV, it is necessary to formulate the correlation between interior and exterior parameters of camera for camera calibration. Interior parameters of camera include characteristics of camera and lens itself, principal point, focal distance and distortion parameters, and exterior parameters of camera include those related to the external environment of camera such as camera rotation and position movement. Information on these parameters can be obtained by a precise optical experiments, or estimated using the geometric relationship between camera and control points.

The normal zoom lens cameras that are currently in use have a lot of merits in acquiring images, but they are geometrically unstable in the course of acquiring real images, and have considerable difficulties in calibrating camera lens due to various zoom movements during the shooting process. Since the camera parameters for calibrating zoom lens are different at each zooming point, the calibration parameters are calculated over various focal distances during the calibration of camera lens, and especially, if the zoom is moved at the time when the lens calibration has already been completed, there are difficulties to recalculate the camera calibration parameters[3,4,5].

Accordingly, this study proposes a comparative analysis on Tsai's camera calibration method and DLT (Direct Linear Transformation) calibration method used to acquire accurate images available for computer vision or photogrammetry that has calibrated zoom lens distortion.

\section{Camera Calibration Method}

When you know the real-world spatial coordinates for any points, you can use camera parameters associated with camera model to calculate the coordinates projected into images for three-dimensional points in the image coordinate system. However, the zoom lens model is difficult to directly apply a fixed focus lens model such as pinhole camera used in a single lens camera, so the individual fixed focus lens model is erected at each discrete point in time, when the zoom movement occurs, to perform calibration. 
Principal point, focal distance, three radial distortion parameters and two tangential distortion parameters can be taken into account as the camera interior parameters, and the camera exterior parameters include rotation matrix and translation vector from the origin of the real-world spatial coordinate to the origin of the camera spatial coordinate.

In order to obtain these cameral parameters, this study conducted a comparative analysis of two camera calibration techniques; Tsai's calibration method and DLT (Direct Linear Transformation) calibration method[6,7].

\subsection{Tsai's Calibration Method}

Figure 1. shows a Tsai camera model assuming parallelism constraint where a 3D spatial coordinate $\mathrm{P}\left(x_{w}, y_{w}, z_{w},\right)$, a coordinate projected on the image by radial distortion $\operatorname{Pd}\left(X_{d}, Y_{d}\right)$, and an image coordinate without radial distortion $\mathrm{Pu}\left(X_{u}, Y_{u}\right)$ meet the parallelism condition at all time like equation (1), regardless of the degree of radial distortion or the distance from the camera.

$$
\overline{O_{i} P d} / / \overline{P_{o z} P} / / \overline{O_{i} P u}
$$

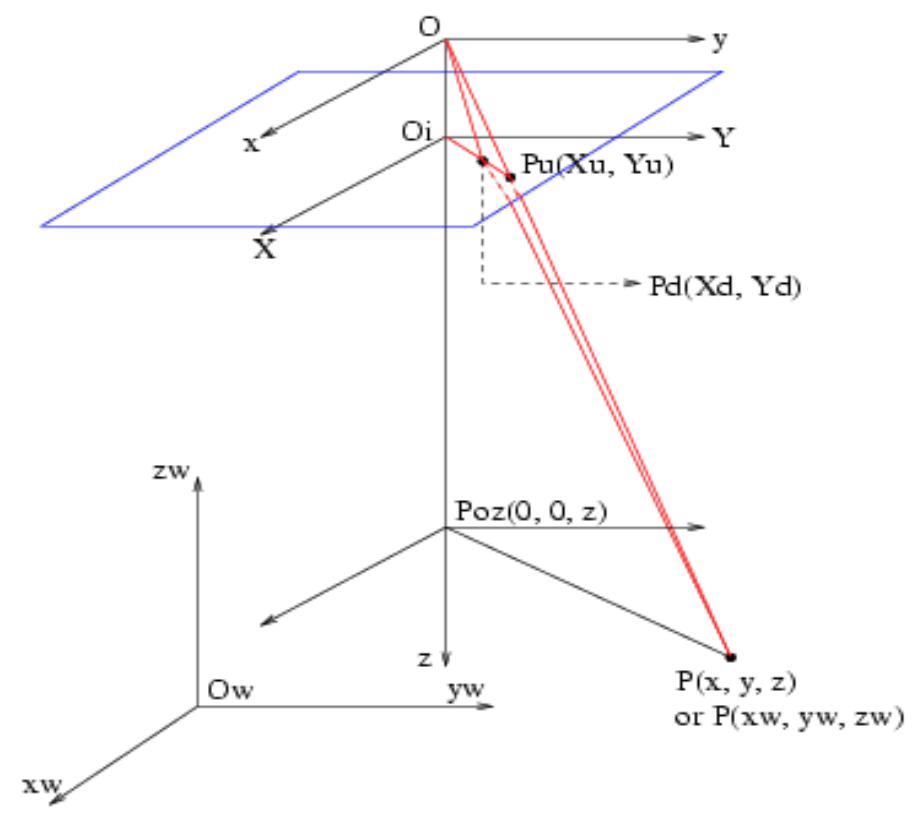

Figure 1 Tsai's Camera Model (Parallelism Constraint)

Tsai's calibration method calculates the camera parameters from a relational equation between control point and the image projection point on the 3D spatial coordinate, based on parallelism constraint in the above equation (1).

\subsection{DLT (Direct Linear Transformation) Calibration Method}

In order to decide theses interior and exterior parameters of camera, this study conducted camera calibration by means of DLT (Direct Linear Transformation) technique based on the collinearity condition that the lens center of camera, the image point in photograph, and the corresponding target point on the actual 3D space must be placed in a straight line.

When you know the real-world spatial coordinate for any points, you can use camera parameters associated with camera model to calculate the coordinates projected into images for three-dimensional points in the image coordinate system. However, the zoom lens model is difficult to directly apply a fixed focus lens model such as pinhole camera used in a single lens camera, so the individual fixed focus lens model is erected at each discrete point in time, when the zoom movement occurs, to perform calibration. Principal points $(X, Y, Z)$, three radial distortion parameters and two tangential distortion parameters can be taken into account as the camera interior parameters, and the camera exterior parameters include translation vector, $x^{\prime}, y^{\prime}$ and $z^{\prime}$ from the origin of the real-world spatial coordinate to the origin of the camera spatial coordinate $[8,9,10]$. In order to obtain these cameral parameters, this study applied a camera calibration technique under DLT (Direct Linear Transformation). 


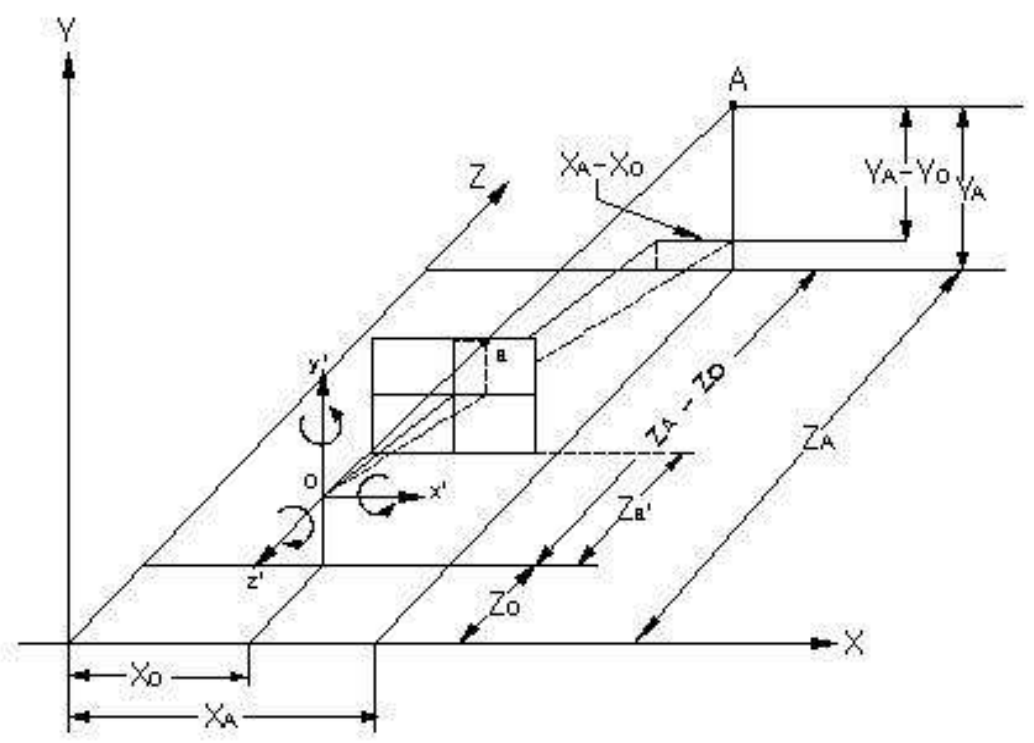

Figure 2 Collinearity condition in the ground photogrammetry

In order to decide theses interior and exterior parameters of camera, this study conducted camera calibration by means of DLT (Direct Linear Transformation) technique based on the collinearity condition that the lens center of camera, the image point in photograph, and the corresponding target point on the actual 3D space must be placed in a straight line. The following Figure 2. shows the concept of the basic collinearity condition in the ground photogrammetry.

\section{3D Restoration applying Tsai's Calibration Method}

Camera parameters obtained through the calibration process in Chapter 2 allow a stereo camera system to be modeled on 3D space as shown in Figure 3.

In such case, 3D spatial coordinate for $P_{1}$ and $P_{2}$, the matching points of the right camera (R) with respect to the left camera (L), can be expressed as coordinates of intersection points for $k_{1} \overrightarrow{R_{1}}$ and $k_{2} \overrightarrow{R_{2}}$, the vectors extended at a certain ratio, $\overrightarrow{R_{1}}=\overline{O_{1} P_{1}} /\left|\overline{O_{1} P_{1}}\right|$ and $\overrightarrow{R_{2}}=\overline{O_{2} P_{2}} /\left|\overline{O_{2} P_{2}}\right|$, the unit vectors for $O_{1}$ and $\mathrm{O}_{2}$ for the optical axis centers of each camera.

However, in general, the vectors, $k_{1} \overrightarrow{R_{1}}$ and $k_{2} \overrightarrow{R_{2}}$, cannot intersect exactly at one spatial coordinate due to the matching errors caused by errors in the resolution of images or calibration parameters. Therefore, taking these errors into account, it is possible to approximate the position of the intersection point at the position where the distance between two vectors, $k_{1} \overrightarrow{R_{1}}$ and $k_{2} \overrightarrow{R_{2}}$, becomes the shortest. In such case, the intersection point, where the distance between two vectors, $k_{1} \overrightarrow{R_{1}}$ and $k_{2} \overrightarrow{R_{2}}$, becomes the shortest, may be assumed to be mid-positioned on the vector $\vec{D}$ perpendicular to both vectors, and the vector $\vec{D}$ perpendicular to both vectors can be expressed as outer products of $\overrightarrow{R_{1}}$ and $\overrightarrow{R_{2}}$ as shown in Equation (2).

$$
\vec{D}=\overrightarrow{R_{1}} \times \overrightarrow{R_{2}}
$$

Consequently, 3D spatial coordinates for the matching points of the two cameras are expressed like the vector equation (3).

$$
k_{1} \overrightarrow{R_{1}}-k_{2} \overrightarrow{R_{2}}+\mathrm{d} \cdot \vec{D}=\vec{b}
$$

Where, $\mathrm{d}$ and $\vec{b}$ represent the length of $\vec{D}$, the vector of intersection point, and the baseline distance vector between the left and right cameras, respectively. 


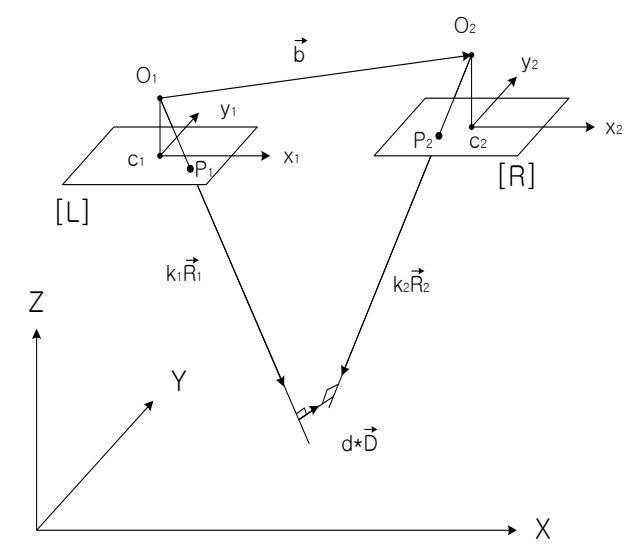

Figure 3 Relationship between parallax and intersection point of stereo model

If we conduct vector analysis on equation (3) again, we can interpret the scale factors, $k_{1}, k_{2}$, and d as shown in equation (4).

$$
k_{1}=\frac{\vec{b} \cdot \vec{D} \times \overrightarrow{R_{2}}}{\overrightarrow{R_{1}} \cdot \vec{D} \times \overrightarrow{R_{2}}}, k_{2}=\frac{\overrightarrow{R_{1}} \cdot \vec{D} \times \vec{b}}{\overrightarrow{R_{1}} \cdot \vec{D} \times \overrightarrow{R_{2}}}, \mathrm{~d}=\frac{\overrightarrow{R_{1}} \cdot \vec{b} \times \overrightarrow{R_{2}}}{\overrightarrow{R_{1}} \cdot \vec{D} \times \overrightarrow{R_{2}}}
$$

Therefore, if equation (4) is put into equation (3), 3D spatial coordinate for the intersection point is expressed as shown in equation (5).

$$
\left[\begin{array}{c}
X \\
Y \\
Z
\end{array}\right]=\left[\begin{array}{c}
X_{O_{1}} \\
Y_{O_{1}} \\
Z_{O_{1}}
\end{array}\right]+k_{1}\left[\begin{array}{l}
R_{1_{x}} \\
R_{1_{y}} \\
R_{1_{z}}
\end{array}\right]+0.5 \mathrm{~d}\left[\begin{array}{c}
D_{x} \\
D_{y} \\
D_{z}
\end{array}\right]
$$

The following Figure 4 shows the flow chart of Direct Linear Transformation(DLT).

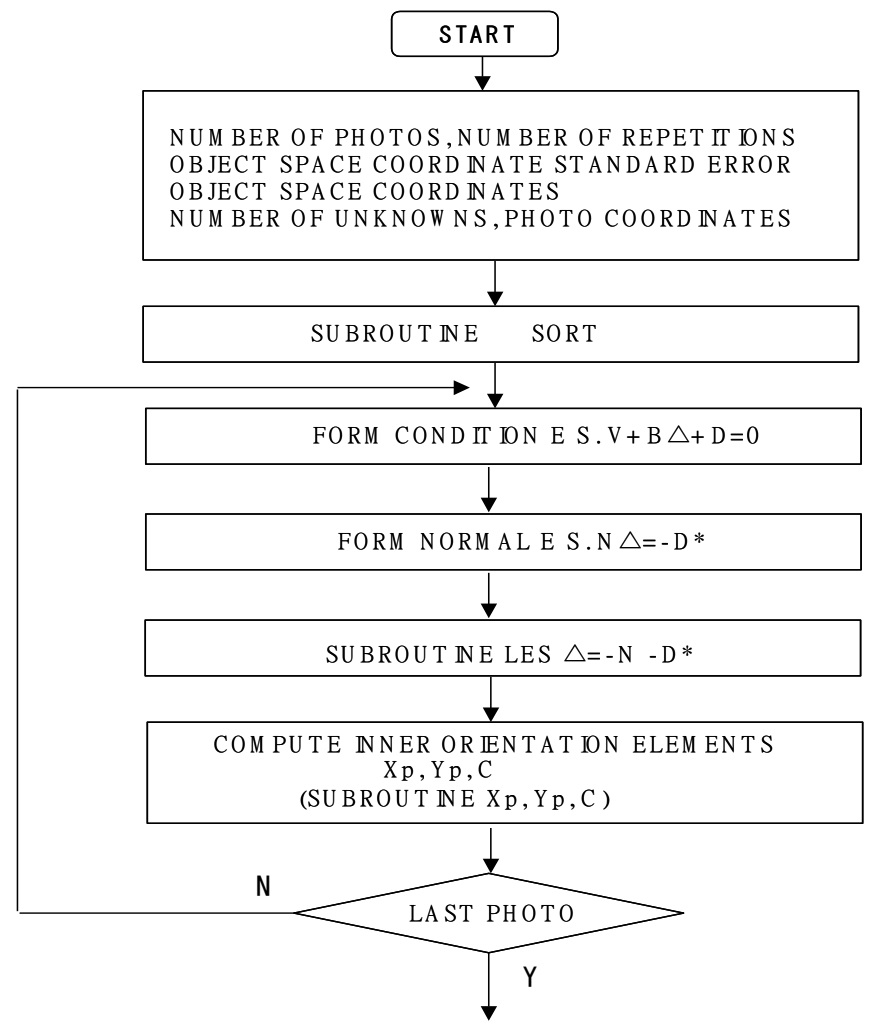




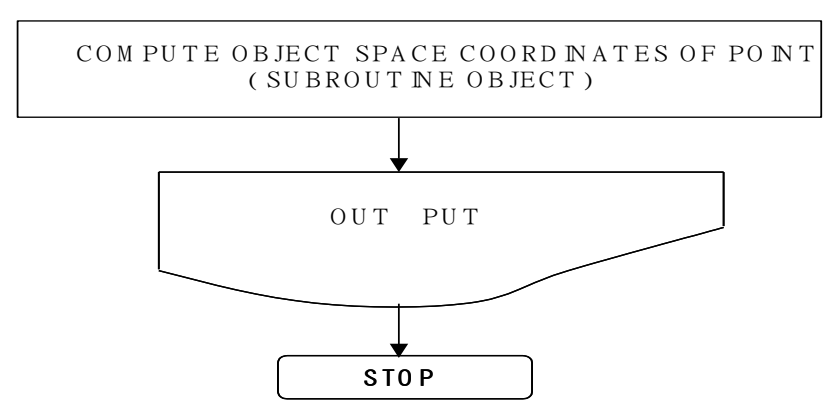

Figure 4 Flow chart of Direct Linear Transformation(DLT)

\section{Analysis of experimental results}

In order to evaluate the restoration performance according to the distance from camera in 3D space, subjects were installed at various distances from camera in the experimental image, and Tsai's calibration method and DLT calibration method were conducted for the acquired images, and the performance of each method were evaluated. Stereo matching and 3D restoration performance were also evaluated using camera parameters obtained from camera calibration.

\subsection{Camera Calibration using Tsai's Coplanar Method}

In order to evaluate the performance of camera calibration using Tsai's coplanar method, this study conducted a camera calibration, making one section of the cubic model in size as a control point, and the performance was evaluated in a manner of restoring cubic model based on camera parameters obtained therefrom. Table 1 shows the calculated value of camera parameters $(\mathrm{mm})$. Table 2 represents the results of error analysis on camera calibration using Tsai's coplanar method.

Table 1 Camera Parameters $(\boldsymbol{m m})$

\begin{tabular}{|l||c|c|c|c|c|c|c|c|c|l|}
\hline & $\mathrm{f}^{1}$ & $\mathrm{k} 1^{2}$ & $T_{x}{ }^{3}$ & $T_{y}$ & $T_{z}$ & $R_{x}{ }^{4}$ & $R_{y}$ & $R_{z}$ & $s_{x}{ }^{5}$ & $C_{x}{ }^{6}, C_{y}$ \\
\hline \hline Left & 17.52 & $-4.63 \mathrm{e}-9$ & -27.34 & 113.97 & 1934.34 & 61.94 & -32.58 & -162.14 & 0.75 & $1003.40,550.46$ \\
\hline Right & 16.48 & $-2.98 \mathrm{e}-9$ & -29.64 & 121.77 & 1819.49 & 102.05 & -35.20 & -162.26 & 0.75 & $960.25,539.88$ \\
\hline
\end{tabular}

Table 2 Results of error analysis on camera calibration using Tsai's coplanar method

\begin{tabular}{|l|l||l|l|l|l|l|l|}
\hline \multicolumn{2}{l||}{ Control Point } & \multicolumn{3}{l|}{ Average of restoration errors } & \multicolumn{3}{l|}{ Standard deviation of restoration errors } \\
\hline Left & Right & $\mathrm{X}$ & $\mathrm{Y}$ & $\mathrm{Z}$ & $\mathrm{X}$ & $\mathrm{Y}$ & $\mathrm{Z}$ \\
\hline \hline 1.225 & 1.239 & 2.945 & 4.128 & 2.874 & 3.417 & 4.877 & 3.446 \\
\hline
\end{tabular}

\subsection{Camera Calibration using Tsai's Non-coplanar method}

In order to the performance of camera calibration using Tsai's non-coplanar method, this study tested two settings. The first is to use cubic model like the coplanar method, and the second is to evaluate the performance of camera calibration using a random control point distributed in the image. Non-coplanar camera calibration was conducted using a cubic model. Like the Coplanar method, camera calibration was

\footnotetext{
$1 \mathrm{f}$ : focal length

2 k1: Radial distortion parameters

${ }^{3} T_{x} T_{y} T_{z}$ : Translation vector

${ }^{4} R_{x} R_{y} R_{z}$ : Rotation matrix

${ }^{5} S_{x}$ : Scale vector

${ }^{6} C_{x} C_{x}$ : Principal coordinate
} 
conducted, making three planes of the cubic model as control points, and the performance was evaluated in a manner of restoring cubic model based on camera parameters obtained therefrom.

Table 3 shows the calculated value of camera parameters $(\mathrm{mm})$. Table 4 represents the results of error analysis on camera calibration using Tsai's non-coplanar method.

Table 3 Camera Parameters ( $\mathrm{mm}$ )

\begin{tabular}{|l||c|c|c|c|c|c|c|c|c|l|}
\hline & $\mathrm{f}$ & $\mathrm{k} 1$ & $T_{x}$ & $T_{y}$ & $T_{z}$ & $R_{x}$ & $R_{y}$ & $R_{z}$ & $s_{x}$ & $C_{x}, C_{y}$ \\
\hline \hline Left & 17.92 & $-5.57 \mathrm{e}-4$ & -17.28 & 137.13 & 1984.99 & 62.54 & -32.97 & -162.38 & 0.75 & $978.13,535.84$ \\
\hline Right & 18.33 & $-1.10 \mathrm{e}-3$ & -7.21 & 138.27 & 2045.35 & 101.79 & -35.96 & -162.13 & 0.76 & $926.34,517.62$ \\
\hline
\end{tabular}

Table 4 Results of error analysis on camera calibration using Tsai's non-coplanar method

\begin{tabular}{|l|l||c|c|c|c|c|c|}
\hline \multicolumn{1}{l||}{ Control Point } & \multicolumn{3}{l||}{ Average of restoration errors } & \multicolumn{3}{|c|}{ Standard deviation of restoration errors } \\
\hline Left & Right & $\mathrm{X}$ & $\mathrm{Y}$ & $\mathrm{Z}$ & $\mathrm{X}$ & $\mathrm{Y}$ & $\mathrm{Z}$ \\
\hline \hline 1.208 & 1.183 & 2.459 & 3.322 & 2.190 & 1.950 & 2.816 & 1.861 \\
\hline
\end{tabular}

As shown in the experimental results, both camera calibrations using Tsai's coplanar method and noncoplanar method show similar results, but the performance of non-coplanar method seems to be slightly superior because the non-coplanar method expresses more information about the space through a random control point.

In conclusion, it can be confirmed that Tsai's camera calibration method affects the result of camera calibration according to the method of setting the control point. However, the error of each method is within a negligible error range depending on the purpose of using the stereo image system. Therefore, the appropriate camera calibration method can be used depending on the purpose.

\subsection{Camera Calibration using DLT Method}

The accuracy of DLT camera calibration was conducted in two aspects as follows. The first was to evaluate accuracy after analyzing the difference between the 3D position estimated from the calculation of model equation and the actually observed 3D position, and the second was to evaluate accuracy in an absolute manner according to the pixel position projected on the image plane in 3D space of the object, that is, the accuracy of the pixel unit.

Table 5 is the result of conducting calibration using the basic DLT coefficients and calculating 3D position, showing that the error from the actual 3D observed value is $1.985 \mathrm{~cm}$ in the $\mathrm{X}$ direction, $12.376 \mathrm{~cm}$ in the $\mathrm{Y}$ direction and $1.283 \mathrm{~cm}$ in the $\mathrm{Z}$ direction.

Table 5 Comparison of 3D positioning results with 11 DLT coefficients and the observed values (unit: $\mathrm{cm}$ )

\begin{tabular}{|l|l|l|l|l|l|l|l|l|l|}
\hline \multirow{2}{*}{$\begin{array}{l}\text { View } \\
\text { points }\end{array}$} & \multicolumn{3}{l}{ Observation points } & \multicolumn{1}{l|}{ Xodeling result points } & \multicolumn{2}{l|}{ Errors } \\
\hline 1 & 60.02 & 610.603 & 56.497 & 54.299 & 630.213 & 59.496 & 5.721 & -19.61 & -2.999 \\
\hline 2 & 89.845 & 620.181 & 56.096 & 88.093 & 626.135 & 56.953 & 1.752 & -5.954 & -0.857 \\
\hline 3 & 119.626 & 615.424 & 56.306 & 119.622 & 611.355 & 55.57 & 0.004 & 4.069 & 0.736 \\
\hline 4 & 164.595 & 624.99 & 55.471 & 164.342 & 612.328 & 53.766 & 0.253 & 12.662 & 1.705 \\
\hline 5 & 209.18 & 615.195 & 55.532 & 211.189 & 634.202 & 58.834 & -2.009 & -19.007 & -3.302 \\
\hline 6 & 238.38 & 617.552 & 55.183 & 239.287 & 620.346 & 55.607 & -0.907 & -2.794 & -0.424 \\
\hline 7 & 269.584 & 610.227 & 55.271 & 275.344 & 630.731 & 58.595 & -5.76 & -20.504 & -3.324 \\
\hline 8 & 89.845 & 620.181 & 31.105 & 91.516 & 608.309 & 29.809 & -1.671 & 11.872 & 1.296 \\
\hline 9 & 239.462 & 620.088 & 30.459 & 241.391 & 629.118 & 31.479 & -1.929 & -9.03 & -1.02 \\
\hline 10 & 59.953 & 610.233 & 6.511 & 60.839 & 607.109 & 6.118 & -0.886 & 3.124 & 0.393 \\
\hline 11 & 89.899 & 620.131 & 6.122 & 92.423 & 603.005 & 5.424 & -2.524 & 17.126 & 0.698 \\
\hline 12 & 119.685 & 615.01 & 6.318 & 117.198 & 632.701 & 6.896 & 2.487 & -17.691 & -0.578 \\
\hline 13 & 164.627 & 624.929 & 5.687 & 164.374 & 663.178 & 6.916 & 0.253 & -38.249 & -1.229 \\
\hline
\end{tabular}




\begin{tabular}{|l|l|l|l|l|l|l|l|l|l|}
\hline 14 & 209.296 & 614.998 & 5.591 & 211.293 & 635.249 & 6.239 & -1.997 & -20.251 & -0.648 \\
\hline 15 & 239.508 & 619.984 & 5.478 & 239.697 & 622.109 & 5.518 & -0.189 & -2.125 & -0.04 \\
\hline 16 & 269.585 & 610.069 & 5.368 & 267.26 & 603.369 & 5.021 & 2.325 & 6.7 & 0.347 \\
\hline 17 & 59.901 & 610.311 & -43.48 & 59.839 & 609.189 & -43.563 & 0.062 & 1.122 & 0.083 \\
\hline 18 & 89.965 & 620.052 & -43.889 & 89.949 & 617.651 & -43.878 & 0.016 & 2.401 & -0.011 \\
\hline 19 & 119.786 & 615.054 & -43.649 & 120.328 & 608.829 & -43.342 & -0.542 & 6.225 & -0.307 \\
\hline 20 & 164.635 & 624.96 & -44.374 & 163.8 & 606.808 & -42.764 & 0.835 & 18.152 & -1.61 \\
\hline 21 & 209.416 & 614.861 & -44.392 & 210.468 & 623.921 & -45.409 & -1.052 & -9.06 & 1.017 \\
\hline 22 & 239.586 & 620.186 & -44.548 & 242.87 & 636.551 & -46.319 & -3.284 & -16.365 & 1.771 \\
\hline 23 & 266.947 & 604.138 & -44.223 & 272.384 & 621.475 & -45.891 & -5.437 & -17.337 & 1.668 \\
\hline 24 & 89.996 & 620.059 & -68.865 & 88.692 & 625.129 & -69.773 & 1.304 & -5.07 & 0.908 \\
\hline 25 & 239.708 & 619.875 & -69.544 & 233.169 & 590.764 & -64.97 & 6.539 & 29.111 & -4.574 \\
\hline Average
\end{tabular}

Table 6 shows the calibration results using the basic 11 DLT coefficients for images taken at a distance of $1 \mathrm{~m}$ from the calibration plate and about $5 \mathrm{~m}$ from the camera, indicating accuracy errors of $1.1 \mathrm{~cm}$ in the $\mathrm{X}$ direction, $62.4 \mathrm{~cm}$ in the depth direction $(\mathrm{Y})$ and $0.77 \mathrm{~cm}$ in the $\mathrm{Z}$ direction.

Table 6 Calibration accuracy for images (distance from calibration plate: $1 \mathrm{~m}, 11$ DLT coefficients)

\begin{tabular}{|c|c|c|c|c|c|c|c|c|}
\hline \multicolumn{3}{|c|}{ Observed Value $(\mathrm{cm})$} & \multicolumn{3}{|c|}{ DLT calculation value $(\mathrm{cm})$} & \multicolumn{3}{|c|}{ Error $(\mathrm{cm})$} \\
\hline $\mathrm{X}$ & $\mathrm{Y}$ & $\mathrm{Z}$ & $\mathrm{X}$ & $\mathrm{Y}$ & $\mathrm{Z}$ & $\Delta \mathrm{X}$ & $\Delta \mathrm{Y}$ & $\Delta \mathrm{Z}$ \\
\hline 165.535 & 467.168 & -13.195 & 164.864 & 542.351 & -13.973 & 0.671 & 75.183 & 0.778 \\
\hline 145.799 & 485.374 & -5.59 & 144.340 & 572.727 & -5.754 & 1.459 & 87.353 & 0.164 \\
\hline 165.677 & 504.569 & 0.386 & 164.617 & 544.880 & -0.31 & 1.06 & 40.311 & 0.696 \\
\hline 185.367 & 486.065 & -7.188 & 186.663 & 569.719 & -8.151 & 1.296 & 83.654 & 0.963 \\
\hline 144.493 & 495.063 & -31.907 & 144.425 & 564.141 & -32.142 & 0.068 & 69.078 & 0.235 \\
\hline 164.346 & 476.734 & -39.527 & 164.002 & 532.851 & -39.542 & 0.344 & 56.117 & 0.015 \\
\hline 184.183 & 495.621 & -33.523 & 181.409 & 520.743 & -31.007 & 2.774 & 25.122 & 2.516 \\
\hline Average & & & & & & 1.10 & 62.40 & 0.77 \\
\hline
\end{tabular}

\section{Conclusion}

This study compared stereo image correction techniques using Tsai's camera calibration method and DLT camera calibration method.

Tsai's coplanar/non-coplanar techniques were able to obtain the reliable interior/exterior parameters of camera in the simulations. DLT method was not able to conduct camera calibration based on a control point existing on a plane, but it was confirmed that a stable camera calibration could be performed at high speeds, taking into account the general environment, such as 3D TV or modeling, and the placement of control point.

Both methods seem to be greatly affected by the performance, depending on the distribution of control points in the image. Therefore, the control points should be evenly placed when using in the actual measurement environment.

Analysis results of camera calibration obtained from this study are thought to be used as reference data for camera calibration when obtaining precise 3D image information in the future.

\section{Acknowledgment}

Funding for this paper was provided by Namseoul University. 


\section{References}

[1] K. He., J. Sun \& X. Tang. Single Image Haze Removal using Dark Channel Prior. Proc. IEEE Conference on Computer Vision and Pattern Recognition, 2009, 1, 1956-1963.

[2] M. Oren \& S. K. Nayar. Generalization of the Lambertian model and implications for machine vision. International Journal of computer vision, 1995, 14(3), 227-251.

[3] Mohamed Benallal \& Jean Meunier. Camera Calibration with a Viewfinder. The 15th International Conference on Vision Interface, 2002 May 27-29, Calgary, Canada.

[4] C. Lawrence Zitnick \& T. Kanade. A Cooperative algorithm for stereo matching and occlusion detection. IEEE Trans. Pattern Anal. Mach. Intell., 2000. 22(7) 675-684.

[5] Blind haze separation, S. Shwartz, E. Namer \& Y. Y. Schechner. Proc. IEEE Conference on Computer Vision and Pattern Recognition, 2006, 2, 1984-1991.

[6] Yong-Shen Chen, Sheng-Wen Shih, Yi-Ping Hung \& Chiou-Shann Fuh. Simple and Efficient Method of Calibrating a Motorized Zoom Lens. Image and Vision Computing, 2001, 19(14), 1099-1110.

[7] J. Salvi, X. Armangue \& J. Batle. A Comparative review of camera calibrating methods with accuracy evaluation. Pattern Recognition, 2002, 35, 1617-1635.

[8] A. Levin, D. Lischinski, \& Y. Weiss.A closed form solution to natural image matting. Proc. IEEE Conference on Computer Vision and Pattern Recognition, 2006, 1, 61-68.

[9] C. Lawrence Zitnick \& T. Kanade. A Cooperative algorithm for stereo matching and occlusion detection. IEEE Trans. Pattern Anal. 2000 Mach. Intell., 22(7), 675-684.

[10] D. J. Jobson, Z. Rahman \& G. A. Woodell. A multiscale retinex for bridging the gap between color images and the human observation of scenes. IEEE Transaction on Image Processing, 1997 July, 6(3), 965-976. 